\title{
UNA TIPOLOGÍA DE CASOS PARA ENSEÑAR LA ADMINISTRACIÓN*
}

\author{
louri Gorbanev**
}

Recibido: octubre 02 de 2012 • Aceptado: diciembre 03 de 2012

\section{RESUMEN}

Objetivo principal de este artículo es proponer una tipología de casos de enseñanza útil para estudiar el impacto del método de casos sobre el aprendizaje. Con base en la teoría de clasificación y la teoría constructivista se diseña la clasificación que sirve para construir la tipología. Se identifican y se caracterizan los tipos ideales de casos. La tipología propuesta está libre de los defectos de las taxonomías existentes y puede ser usada en los estudios de impacto del método de casos sobre el aprendizaje.

\section{PALABRAS CLAVE}

Método de casos, tipología, taxonomía, enseñanza de administración

\section{CLASIFICACIÓN JEL}

A22, M10

CONTENIDO

Introducción; 1. Literatura; 2. Método; 3. Clasificación y tipología; 4. Discusión y conclusiones; Bibliografía

\footnotetext{
Es un trabajo de reflexión que surge a raíz de las dificultades con la evaluación del efecto del método de casos en la enseñanza de Administración.

** Periodista internacional, MGIMO, Moscú, Rusia, 1978. Maestría en Economía, Pontifica Universidad Javeriana, Bogotá, Colombia, 2000. Profesor, Departamento de Administración, Pontificia Universidad Javeriana. Grupo de estudios de Dirección Estratégica y Organizaciones clasificado B en Colciencias. Cra. 8 No. 40-62, Ed. Central, p. 4, Bogotá, Colombia. Tel . Tel. 3208320. Correo electrónico: yurigor@ javeriana.edu.co.
} 
louri Gorbanev

\section{A CASE TYPOLOGY FOR TEACHING ADMINISTRATION}

\section{ABSTRACT}

The main objective of this article is to propose a case typology for the useful teaching for studying the case method impact over teaching. Based on the classification and constructivism theories, a classification useful for constructing a typology is designed. The ideal case types are identified and characterized. The proposed typology is free from defects that existent taxonomies have and can be used in the impact studies for the learning case method.

\section{KEY WORDS}

Case method, typology, taxonomy, business management teaching

\section{JEL CLASSIFICATION}

A22, M10

\section{CONTENT}

Introduction; 1. Literature; 2. Method; 3. Classification and typology; 4. Discussion and conclusions; Bibliography.

\section{UMA TIPOLOGÍA DE CASOS PARA ENSINAR A ADMINISTRAÇÃO}

\section{RESUMO}

O objetivo principal deste artigo é propor uma tipologia de casos de ensino útil para estudar o impacto do método de casos sobre o aprendizado. Com base na teoria da classificação e a teoria construtivista se desenha a classificação que serve para construir a tipologia. Identificam-se e caracterizam-se os tipos ideais de casos. A tipologia proposta esta livre dos defeitos das taxonomias existentes e pode ser usada nos estudos de impacto do método de casos sobre o aprendizado.

\section{PALAVRAS CHAVE}

Método de casos, tipologia, taxonomia, ensino de administração

CLASIFICAÇÃO JEL

A22, M10

\section{CONTEUDO}

Introdução; 1. Literatura; 2. Método; 3. Classificação e tipologia; 4. Discussão e conclusões; Bibliografia 
Una tipología de casos para enseñar la administración

\section{INTRODUCCIÓN}

Método de casos (MC) es una estrategia pedagógica cuyo uso en la enseñanza de administración va en aumento. A pesar de su popularidad, persisten dudas en cuanto al efecto que ejerce el método de casos. Los estudios de su impacto sobre el aprendizaje no producen resultados coherentes porque tienen un defecto metodológico: tratan los casos de estudio como objetos homogéneos. En la realidad los casos no son homogéneos y se diferencian por su volumen, tema, objetivo pedagógico, forma de presentación y redacción del material. Si se quiere entender el impacto de MC sobre el aprendizaje, hay que aplicar el método científico cuyo primer paso es la clasificación.

Varios autores intentaron responder a esta necesidad con clasificaciones de casos. McNair (1971, p. 431), citado por Rippin y otros (2002, p. 431), distingue dos clases: casos para analizar y casos para tomar decisiones. Leenders y Erskine (1989), citado por Rippin y otros (2002, p. 431), sugieren tres dimensiones para clasificar casos: dificultad, tarea analítica que plantean ante el estudiante, volumen y claridad del caso. Heath (1997), citado por Rippin y otros (2002, p. 431), propone seis clases que van de casos cortos que narran un incidente hasta casos complejos de decisión. La clasificación de Lundberg y otros (2001) se basa en una variedad de criterios entre los cuales figuran la naturaleza y cantidad de la información utilizada, actividad que el alumno debe desarrollar y otros sin un enfoque teórico determinado.

El problema de estas taxonomías es que no están fundamentadas en la teoría por lo cual no se usan por los investigadores de impacto.

Ellet (2007, p. 20-26) dio un paso para crear una clasificación fundamentada en la teoría y propuso cuatro clases de casos: problema, evaluación, toma de decisiones, aplicación de algoritmo. Su clasificación está basada en la taxonomía de problemas de Jonassen (2000, pp. 74-76). Pero la naturaleza del problema es solo un atributo del caso. Por otro lado, el autor no ofrece ventaja al reducir el número de categorías de Jonassen (2000) de 10 a 4. Por ejemplo, Ellet (2007) descarta el diseño como categoría mientras la literatura gerencial identifica el diseño como la actividad gerencial por excelencia. La frontera entre los casos tipo problema y tipo evaluación está poco clara. El mérito de Ellet (2007) es haber utilizado la categorización de problemas para clasificar los casos. De esta manera quedó establecido que el grado de estructuración del problema es uno de los atributos del caso.

Las clasificaciones existentes no están basadas en la teoría. Por esta razón las tipificaciones no fueron aceptadas por la comunidad y ni siquiera existe una terminología común para referirse a los atributos de los casos. 
El objetivo de este trabajo es proponer una tipología de casos fundamentada en la teoría constructivista.

Con base en la teoría de clasificación y la teoría constructivista se identifican los ejes conceptuales para construir la clasificación y tipología. Se identifican y se caracterizan los tipos ideales de casos.

La tipología propuesta es útil por dos motivos. Primero, porque proporciona un lenguaje común para discutir los atributos de los casos entre los profesores y autores. Segundo, porque permite evitar hablar del efecto del método de casos en abstracto y concentrarse en el impacto de un tipo determinado de casos sobre el aprendizaje.

En lo que sigue, el trabajo se divide en cuatro secciones: resumen de literatura, método, clasificación y tipología, discusión y conclusiones, seguidas de la bibliografía.

\section{LITERATURA}

Se utilizan dos corrientes literarias: la teoría de clasificación y la constructivista que describen y explican el proceso de aprendizaje.

La literatura sobre la clasificación inicia con los filósofos griegos. Parménides propuso el principio de separación entre dos clases de objetos basada en la diferencia: si unos objetos pertenecen a la clase A, no pertenecen a las clases No-A. Todos los objetos en A comparten las características presentes en la definición de concepto de A. Según Fox (2011, p. 329), Platón propuso tres leyes de clasificación: no contradicción (ningún objeto puede estar en la clase A y en No-A); de identidad (lo que esta en A, pertenece a A) y la ley de exclusión de grado medio (todo objeto debe estar en A o en No-A).

La teoría clásica establece que la clasificación debe ser exclusiva: un objeto no puede pertenecer a más de una clase y debe estar en alguna. En la práctica resulta difícil crear clases mutuamente excluyentes porque los objetos suelen combinar varias características en distintos grados de intensidad y no obedecen al patrón sí-no. La clasificación pierde la pureza binaria y se vuelve asunto de grado en que un objeto ostenta características de diferentes clases. De acuerdo con Fox (2011, p. 329-330), las clasificaciones se vuelven un ordenamiento a lo largo de unos ejes.

Criag (1998, p. 272) plantea que una buena clasificación agrupa entidades en clases de tal manera que las clases facilitan la formulación de las leyes científicas. Cuando las características de la clase no solo sirven para definir las casillas que 
Una tipología de casos para enseñar la administración

acomodan objetos sino contribuyen a hacer generalizaciones y predicciones, se trata de tipología (Weber, 1949; Karlovskaya, 2012; Tong y otros, 2008).

La tipología está relacionada con la construcción de tipos ideales. El concepto que determina la clase no se considera como una fiel representación de todos los objetos en esa clase sino como un tipo ideal.

Al respecto Weber (1949, p. 90) plantea:

El tipo ideal se forma mediante el énfasis unilateral en ciertos puntos de vista y mediante la síntesis de los numerosos fenómenos individuales que son difusos, discretos, mas o menos presentes y a veces ausentes y que están organizados en un constructo analítico unificado. Este constructo mental no se puede encontrar en la realidad en su pureza conceptual. Es una Utopía.

Si bien el tipo ideal no existe, es fundamental en las ciencias sociales porque hace clara no tanto la clase a que pertenece el fenómeno, ni el carácter promedio del fenómeno sino su carácter único, y permite ver su importancia práctica (Weber, 1949, p. 102).

La segunda corriente literaria está representada por el constructivismo que postula que el aprendizaje no es otra cosa sino la búsqueda del significado, y que la persona construye su propio conocimiento sobre el mundo. El conocimiento es construido en el proceso de la interacción con el ambiente social y físico. En el fundamento del constructivismo se encuentran los estudios de Piaget (1961) y Vygotsky (1986). Según Vygotsky (1986), el concepto científico no es una formación aislada y osificada sino una parte activa del proceso intelectual que sirve a la comprensión, comunicación y solución de problemas. La formación del concepto científico en el alumno no es un proceso mecánico. El saber se apropia por el alumno en curso de una operación compleja dirigida hacia la solución de algún problema. La mera vinculación mecánica entre la palabra y el objeto no es suficiente para producirlo. De aquí los constructivistas concluyen que el alumno es responsable de su propio proceso de aprendizaje.

Coll (2000) llama la atención a una paradoja en la teoría. Si el alumno puede aprender solamente lo que es capaz de construir, ¿cómo se puede enseñarle algo?, ¿en qué consiste el rol del profesor? La teoría contesta esta pregunta en términos generales: el profesor crea las situaciones que favorecen el despliegue de la actividad mental del alumno y guía al alumno en la dirección que señalan los saberes seleccionados como objetivos de aprendizaje. Por eso no existe la metodología didáctica constructivista sino una estrategia didáctica general de naturaleza constructivista que se concreta en múltiples formas didácticas según el caso. A veces se da al alumno 
información organizada; a veces, modelos de acción, en otra ocasión, se formulan indicaciones sobre cómo resolver tareas; en algunos casos se permite que el alumno desarrolle de manera autónoma determinadas actividades. El profesor, capaz de promover el aprendizaje en sus alumnos, es el que puede utilizar de forma flexible una amplia gama de recursos didácticos (Coll, 2000, p. 26). Es imposible resolver la paradoja mencionada de manera más concreta porque nuestro conocimiento sobre cómo los profesores consiguen ajustar su ayuda al proceso de construcción de conocimiento está limitado (Coll, 2000, p. 26).

Wu y otros (2011) hacen un resumen de los enfoques educativos agrupados bajo el paraguas de constructivismo: teoría de desarrollo social, método de casos, aprendizaje cognoscitivo, aprendizaje por descubrimiento, aprendizaje basado en problemas, aprendizaje situado, teoría de actividad, teoría de red-actor, a los cuales se podría agregar la teoría de aprendizaje significativo. Ausubel, Novak y Hanexian (1983, p. 308) responden a la preocupación de Coll (2000) y formulan algunas características del material didáctico para el aprendizaje significativo. Según los autores, tal material debe expresar los objetivos de aprendizaje de manera clara y relacionar el nuevo saber con el saber ya existente en el alumno. El material debe ser motivante porque la motivación es el mecanismo que permite al alumno relacionar el saber existente con el nuevo (Ausubel, Novak y Hanexian, 1983, p. 352-354). El material debe tener un tamaño razonable porque su dimensión determina la dificultad de la tarea y la motivación del alumno (Ausubel, Novak y Hanexian, 1983, p. 322).

La teoría constructivista gira en torno a la solución de problemas. Los problemas se ofrecen a los alumnos en forma de preguntas. La taxonomía clásica de preguntas pertenece a Bloom (1986, p. 127-131) quien distribuye las preguntas posibles en seis niveles de actividad mental:

1. Conocimiento. Una pregunta de conocimiento pide al alumno reproducir la información de un texto.

2. Comprensión. Una pregunta de este tipo pide al alumno darle otra presentación a la información dada en un texto.

3. Aplicación. Una pregunta tipo aplicación pide al alumno aplicar los hechos, principios y teorías para solucionar un problema.

4. Análisis. Una pregunta de análisis pide al alumno identificar tendencias en series de eventos y procesos.

5. Síntesis. Una pregunta tipo síntesis invita al alumno a pensar de manera creativa para generar una explicación o solución original de una situación. 
Una tipología de casos para enseñar la administración

6. Evaluación. Una pregunta de evaluación pide al alumno hacer un juicio ético y determinar si un hecho, idea o concepto está de acuerdo con ciertos estándares y valores.

Jonassen (2000, p. 74-76) ofrece una clasificación de problemas en el continuo de problemas estructurados-no estructurados: problemas lógicos tipo adivinanzas; problemas algorítmicos que exigen la aplicación de un juego limitados de reglas, como los problemas matemáticos; problemas algorítmicos que están acompañados por una historia; problemas que exigen el uso de múltiples reglas para solucionarlos, como ajedrez; toma de decisión, o la elección de una opción con base en unos criterios; la corrección de la falla a través del diagnóstico y la eliminación de la causa de la falla; problemas estratégicos que exigen no solo la aplicación de unos principios sino también la improvisación y la generación de nuevas herramientas, reglas y principios; estudio de caso que implica la solución de un problema no estructurado donde no se establece qué es una buena solución y la información disponible es incompleta o ambigua; problemas de diseño que implican una formulación ambigua del objetivo, la ausencia de las reglas y la necesidad de conocimiento multidisciplinar; dilema que no tiene una solución aceptable para todas las personas a causa de diferencias en sus valores.

\section{MÉTODO}

Se sigue a Bailey (1973, p. 293-294) para construir la tipología heurística politética. La tipología propuesta va a ser heurística, es decir, basada en los tipos ideales. También va a ser politética, es decir, va a agrupar los casos y problemas (CP) con el mayor número de características comunes sin pretender crear grupos exclusivos.

Con base en la literatura constructivista se identifican los ejes pertinentes para clasificar los casos. La clasificación se entiende como el ordenamiento de objetos en grupos con base en sus relaciones, cercanía y similitud. La clasificación sirve para identificar, entre todas las clases posibles, aquellas clases de casos que más contribuyen al aprendizaje. Acto seguido estas clases se relacionan con los tipos ideales.

\section{CLASIFICACIÓN Y TIPOLOGÍA}

Los ejes para clasificar los casos los sugiere la teoría constructivista. Para que el aprendizaje ocurra, el alumno tiene que enfrentar un problema. En el mundo real, el problema ocurre de manera natural. En el mundo artificial del aula, el problema tiene que estar formulado por el profesor como un caso. Para cumplir su función de provocar la actividad constructora mental del alumno, el caso tiene que ser 
motivador: simular la realidad, plantear un reto, representar una organización importante, describir una historia no trivial. Al contarla, el caso debe ser corto para no desmotivar al alumno. Los casos sirven para desencadenar el esfuerzo del alumno dirigido a la solución del problema. La intensidad de su esfuerzo depende del grado de estructura que ofrece el caso.

Se pueden representar las tres dimensiones en forma de un cubo, como en la gráfica 1.

Grafica 1. Tres ejes para ordenar los casos y problemas.

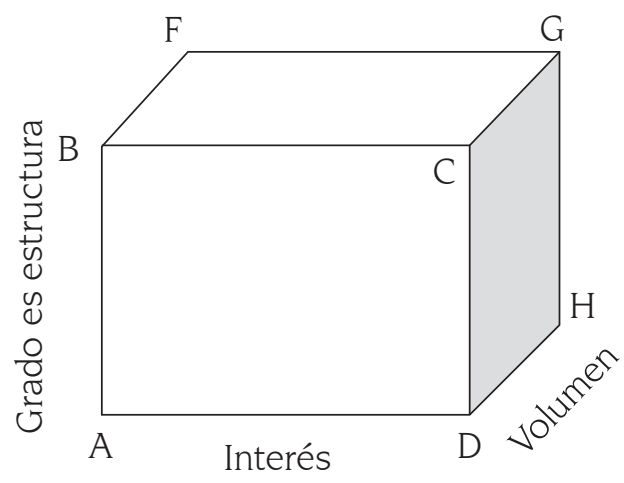

Fuente: diseño del autor

Surgen 8 clases de casos ubicadas en las esquinas del cubo. La esquina A sirve como el origen.

- A: Casos de poco interés, cortos y no estructurados

- B: Casos de poco interés, cortos y estructurados

- C: Casos de alto interés, cortos y estructurados

- D: Casos de alto interés, cortos y no estructurados

- E (oculta): Casos de poco interés, largos y no estructurados

- F: Casos de poco interés, largos y estructurados

- G: Casos de alto interés, largos y estructurados

- H: Casos de alto interés, largos y no estructurados

Los casos atractivos, que van a gustar a los alumnos, son cortos e interesantes, ubicados en el eje CD. ¿Qué tipos ideales están ubicados en el eje CD? Para 
Una tipología de casos para enseñar la administración

identificarlos, se acude a la taxonomía de Jonassen (2000, p. 74-76). El primer tipo ideal es algoritmo: un caso corto y pragmático que no pierde tiempo en describir organizaciones glamorosas ni crear fábulas atractivas. Cualquier organización real o ficticia es buena para plantear la situación problemática que es el centro del algoritmo. El problema representado en ese caso está estructurado, por lo cual el profesor no admite opiniones infundadas por parte del alumno y exige el dominio de la herramienta administrativa determinada. El algoritmo tiene una sola respuesta correcta y un solo procedimiento correcto que el alumno está obligado a seguir. El algoritmo pone a prueba la capacidad del alumno de diagnosticar la situación y detectar la necesidad de aplicar la herramienta administrativa determinada. También pone a prueba el conocimiento de la herramienta, como por ejemplo, de una fórmula matemática. El algoritmo permite evaluar la capacidad del alumno de aplicar la herramienta administrativa para obtener el único resultado correcto.

El tipo ideal de ajedrez, que se ubicaría en la mitad del eje CD, es un caso que presenta una fábula bastante atractiva para involucrar al alumno en la solución del problema. El caso tipo ajedrez se enfoca en organizaciones y personajes reales relevantes para el lector y plantea un problema real que es o fue objeto de discusión en los medios y la sociedad en general. La organización descrita en el caso experimenta un problema que el lector está invitado a resolver. Al narrar el problema, el autor del caso diseña las restricciones que obligan al alumno a utilizar cierta teoría y modelo administrativo. El ajedrez pone a prueba dos aspectos del aprendizaje: la capacidad del alumno de diagnosticar la situación y escoger el modelo administrativo adecuado y, segundo, su capacidad de aplicar el modelo seleccionado para solucionar el problema. El caso tipo ajedrez no tiene una solución única y el alumno tiene la libertad para formular su propuesta, siempre y cuando sigue la teoría adecuada y no viola las restricciones establecidas.

Ajedrez no sirve para memorizar conceptos y se ubica en los niveles de aplicación, análisis y síntesis de Bloom (1986, p. 127-131) y es fundamental para generar las habilidades gerenciales.

El tipo ideal bestseller ocupa el extremo D del eje CD. Es complejo, no estructurado y permite al alumno opinar, plantear hipótesis y proponer soluciones sin restricciones, por lo cual ayuda a generar capacidades de análisis, síntesis y evaluación. En cambio, bestseller es poco útil para entrenar al alumno en la aplicación de una teoría o de un modelo administrativo. Más aún, bestseller deja abierta la posibilidad de no utilizar ningún modelo sino limitarse al sentido común. El profesor que utiliza CP tipo bestseller tiene dificultades a la hora de evaluar la calidad de aporte del alumno en la discusión del caso.

Semestre Económico, volumen 15, №. 32, pp. 185-196 • ISSN 0120-6346, julio-diciembre de 2012, Medellín, Colombia 
louri Gorbanev

La tipología no puede limitarse a identificar los tipos ideales deseables para el profesor sino debe identificar los tipos de casos con una identidad inconfundible. En este sentido es evidente la existencia de casos largos que representan un bloque importante de esos documentos y tienen una fisonomía propia. Telenovela es el tipo ideal de casos largos que traen una amplia información sobre el sector industrial, la historia de la organización, nombres y cargos de numerosos personajes. El lector se pierde en detalles, nombres y fechas cuya importancia para la solución del problema no es evidente. Telenovela puede tener una fábula interesante pero la extensión del texto genera la desmotivación del alumno. Una vez perdido el interés, el proceso de aprendizaje se frena. Este fenómeno se acentúa cuando el texto viene en un idioma extranjero. El profesor que utiliza casos tipo telenovela tiene dificultades a la hora de captar la atención del alumno y centrar la discusión en temas clave para el aprendizaje. Sin embargo, los casos tipo telenovela se encuentran con frecuencia porque los autores consideran necesario incluir en sus documentos el "ruido" informativo, so pretexto de que en la vida real el problema viene disfrazado de hechos y datos que distraen al gerente. Es difícil estar de acuerdo con este planteamiento porque un caso es una creación literaria y es juzgada como tal por sus lectores que resisten absorber datos irrelevantes.

\section{DISCUSIÓN Y CONCLUSIONES}

La tipología propuesta permite abrir la caja negra del método de casos. En vez de prueba y error el profesor puede utilizar la tipología propuesta para seleccionar los casos que mejor sirven para sus propósitos. Si se necesita que los alumnos memoricen y comprendan los conceptos, modelos y teorías, lo aconsejable son casos de tipo algorítmico. Si el profesor quiere ejercitar las capacidades de aplicación y análisis, tiene a su disposición los casos tipo ajedrez. Cuando el profesor necesita construir las capacidades de síntesis y evaluación, puede dirigirse a los casos tipo bestseller que se ajustarán mejor a estos niveles altos de actividad mental. Para combatir el tedio y la baja participación, el profesor identificará los casos tipo telenovela para evitarlos.

Para el investigador que trata de determinar el impacto del método de casos, la tipología hace evidente que no existen los casos en general, por lo cual pierde sentido hablar sobre el efecto del método de casos sobre el aprendizaje. Pero se puede hablar sobre el efecto que ejerce sobre el aprendizaje cierto tipo de casos.

Para un autor de casos, la tipología le hace evidente dos cosas: un caso es una obra literaria para la cual no existe patrón y, segundo, ningún tipo de casos puede asegurar el logro de todos los objetivos de aprendizaje. 
Una tipología de casos para enseñar la administración

Para la comunidad de profesores que utilizan los casos, la tipología propuesta proporciona una terminología común que facilita el dialogo entre profesores y permite comparar sus experiencias.

La debilidad de este trabajo consiste en la ausencia de una prueba empírica. Para hacer tal prueba, hay que determinar la distancia que separa unos casos concretos de su tipo ideal y medir el efecto diferencial que esos casos tienen sobre el aprendizaje.

\section{BIBLIOGRAFÍA}

Ausubel, D., Novak, J., y Hanesian, H. (1983). Psicología educativa. México: Trillas, 623p.

Bailey, K. (1973). Monothetic and Polythetic Typologies and their Relation to Conceptualization, Measurement and Scaling. En: American Sociological Review, Vol. 38, N. 1 (Jstor), pp. 18-33.

Bloom, B. (1986). Taxonomía de objetivos de educación. Buenos Aires: Ateneo, 273p.

Coll, C. (2000). Constructivismo e intervención educativa, pp. 11 -32. En Barbera, Elena (2000). El constructivismo en la práctica. Caracas: Laboratorio educativo, 157p.

Craig, E. (1998). Routledge encyclopedia of philosophy. London: Routledge.

Ellet, W. (2007). The case study handbook. Boston, Mass.: Harvard Business School Press, 272p.

Fox, M. (2011). Prototype Theory: An Alternative Concept Theory for Categorizing Sex and Gender? En: Knowledge Organization, N. . 38, pp. 328-340.

Heath, J. (1997). Teaching and writing case studies. Bedford: ECCH.

Jonassen, D. (2000). Toward a design theory of problem solving. En: Educational Technology, Research and Development, Vol. 48, N. ${ }^{\circ}$ 4, pp. 63-85.

Karlovskaya, E. (2012). Typology and Systematization of State Services. En: Bulletin of PNU, Vol. 24, N.․ 1, pp. 179-189.

Leenders, M. y Erskine, J. (1989). Case research: the case writing process. London, Ontario: University of Wenster Ontario.

Lundberg, C.; Rainsford, P.; Shay, J. y Young, C. (2001). Case writing reconsidered. En: Journal of Management Education, Vol. 25, N. ${ }^{\circ}$ 4, pp. 450-463.

McNair, M. P. (1971). McNair on cases. En: Harvard Business School Bulletin, julio-agosto, ECCH Reprint N. ${ }^{\circ}$ 9, pp. 372-303.

Piaget, J. (1961). La formación del símbolo en el niño. México: FCE, 401p. 
Rippin, A.; Booth, C.; Bowie, S. y Jordana, J. (2002). Complex Case: using the case study method to explore uncertainty and ambiguity in undergraduate business education. En: Teaching in Higher Education, Vol. 7, N.․ 4, pp. 429-441.

Tong, P.; Johnson, J.; Umesh, U. y Ruby, P. (2008). A typology of interfirm relationships: the role of information technology and reciprocity. En: The Journal of Business $\&$ Industrial Marketing, Vol. 23, N. 3 , pp. 178-192.

Vygotski, L. (1986). Thought and language. Cambridge, Mass: The MIT Press, 287p.

Weber, M. (1949). The Methodology of the Social Sciences. Glencore, Ill.: Free Press, 183p.

Wu, W., Hsiao, H., Wu, P., Lin, C., \& Huang, S. (2011). Investigating the learning-theory foundations of game-based learning: a meta-analysis. En: Journal of Computer Assisted Learning, Vol. 28, N. ${ }^{\circ}$ 3, pp. 265-279. 